Ann. Parasitol. Hum. Comp., 1992, $67: \mathrm{n}^{\circ} 6,188-193$.

Mémoire.
Mots-clés : Gongylonema (Progongylonema) n. subgen. Corvidae. Spain. Gongylonema (Progongylonema) pacoi n. sp.

Key-words: Gongylonema (Progongylonema) n. subgen. Corvidae. Spain. Gongylonema (Progongylonema) pacoi n. sp.

\title{
GONGYLONEMA (PROGONGYLONEMA) PACOI N. SUBGEN. N. SP. (SPIRUROIDEA : GONGYLONEMATIDAE) PARASITE D'OISEAUX CORVIDAE
}

\author{
S. HERNANDEZ-RODRIGUEZ, P. N. GUTIÉRREZ-PALOMINO
}

\begin{abstract}
RÉSUMÉ
Description de Gongylonema (Progongylonema) pacoi n. subgen. n. sp. Nématode parasite de la muqueuse buccale de Pica pica, Garrulus glandarius, Cyanopica cyanus et Corvus monedula (Passeriformes : Corvidae) dans la zone nord de la province de Cordoue (sud de l'Espagne). La structure céphalique apparente l'espèce aux Gongylonematidae. Gongylonema (Progongylonema) diffère
\end{abstract}

essentiellement des sous-genres Gongylonema (Gongylonema) et Gongylonema (Gongylonemoides) par l'absence d'écussons cuticulaires. Gongylonema (Progongylonema) pacoi n. sp., est caractérisé par la présence de deirides et d'ailes latérales. Mâle avec les spicules très inégaux, gubernaculum présent et cloaque proéminent avec un rebord festonné.

Summary: Gongylonema (Progongylonema) pacoi n. subgen n. sp. (Spiruroidea: Gongylonematidae) parasite of Corvidae.

A new Nematode Gongylonema (Progongylonema) pacoi n. subgen. n. sp. parasite from the mucosa under the tongue of Pica pica, Garrulus glandarius, Cyanopica cyanus and Corvus monedula (Passeriformes: Corvidae) from north of Córdoba province (Southern Spain) is described. Because of its cephalic structure the species is considered as Gongylonematidae. Gongylonema
(Progongylonema) is distinguished mainly from the subgenera Gongylonema (Gongylonema) and Gongylonema (Gongylonemoides) by the absence of cuticular bosses. Gongylonema (Progongylonema) pacoi $\mathrm{n}$. sp. is characterised by showing deirids and lateral alae. Males with spicules very unequal, gubernaculum present and prominent cloaca with a festooned border.

\section{MATÉRIEL ET MÉTHODES}

Les observations réalisées sur 275 pies (Pica pica), 28 geais (Garrulus glandarius), 65 pies bleues à calotte noire (Cyanopica cyanus) et 55 choucas des tours (Corvus monedula) (Passeriformes : Corvidae) capturés dans la zone nord de la province de Cordoue (Val des Pedroches), ont révélé une prévalence assez élevée de spiruridés dans la muqueuse de la partie inférieure de la cavité buccale. Le nombre d'exemplaires obtenus à partir d'hôtes étudiés a été de 236 (83 mâles et 153 femelles). Le matériel étudié correspond à 1 mâle holotype, 1 femelle allotype, 9 mâles paratypes et 9 femelles paratypes, déposés dans la collection parasitologique du Département de Parasitologie de la Faculté Vétérinaire de l'Université de Cordoue avec les numéros "HA-1092 mâle holotype », « HA-1092 femelle allotype", "HA-1092 mâles paratypes " et " HA-1092 femelles paratypes".

Les exemplaires obtenus sont lavés au sérum physiologique, fixés dans l'alcool-glycérine, montés et éclaircis au lactophénol pour leur étude au microscope.

\section{DESCRIPTION}

\section{Hôte :}

Pie (Pica pica); Geai (Garrulus glandarius); Pie bleue à calotte noire (Cyanopica cyanus) et Choucas des tours (Corvus monedula).

Departamento de Parasitologia y Enfermedades Parasitarias, Facultad de Veterinaria, Universidad de Córdoba, 14071 Córdoba, Spain.

Accepté le : 17 mars 1992.

\section{Localisation :}

Muqueuse de la partie inférieure de la cavité buccale.

\section{Distribution géographique :}

Val des Pedroches (zone nord de la province de Cordoue, Espagne).

\section{Prévalence :}

16 des 275 pies $(6 \%)$

11 des 65 pies bleues à calotte noire $(17 \%)$

6 des 28 geais $(21 \%)$

5 des 55 choucas des tours $(9 \%)$.

\section{Intensité :}

Pie (Pica pica) : 5 à 9 exemplaires par hôte.

Pie bleue à calotte noire (Cyanopica cyanus) : 2 à 8 exemplaires par hôte.

Geai (Garrulus glandarius) : 4 à 8 exemplaires par hôte.

Choucas des tours (Corvus monedula) 3 à 7 exemplaires par hôte.

Ouverture buccale limitée intérieurement par deux formations latérales trilobulées et deux élévations dentiformes sclérotisées (l'une dorsale et l'autre ventrale) qui dépassent la bouche. Rebord sclérotisé quadrangulaire étiré dorsoventralement avec des angles émoussés et élevé en forme 
de collier. Trois paires de papilles labiales internes disposées en deux groupes latéraux et en ligne, quatre papilles labiales externes disposées dans les angles émoussés du rebord et un cercle externe de quatre papilles céphaliques. Deux amphides en contact avec la marge latérale du rebord. Deirides situées en avant de l'anneau nerveux. Ailes latérales, de longueur différente chez la femelle et chez le mâle, débutant toujours en arrière des deirides. Cuticule striée transversalement et sans écussons cuticulaires. Pharynx cylindrique à parois minces. Csophage long et divisé en deux portions.
FIG. 1. - Gongylonema (Progongylonema) pacoi $\mathrm{n}$. sp. Mâle.

A : extrémité antérieure. B : extrémité postérieure. $\mathrm{C}$ : détail du cloaque. $\mathrm{D}$ : gubernaculum, vue latérale. E : gubernaculum, vue ventrale. $\mathrm{F}$ : extrémité antérieure du spicule droit. $\mathrm{G}$ : spicule gauche. $\mathrm{H}$ : extrémité antérieure, vue ventrale. I : région antérieure, vue ventrale.
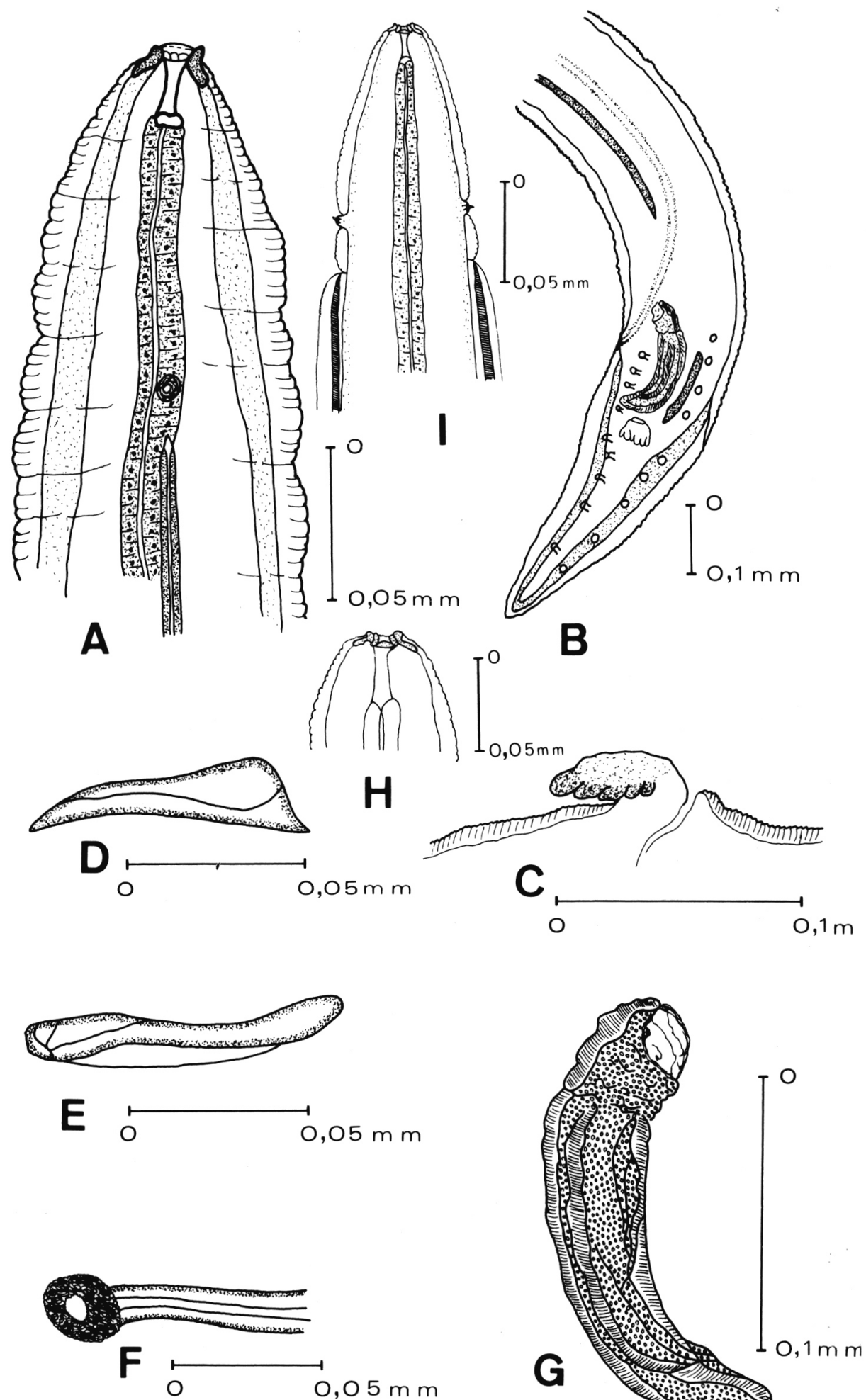

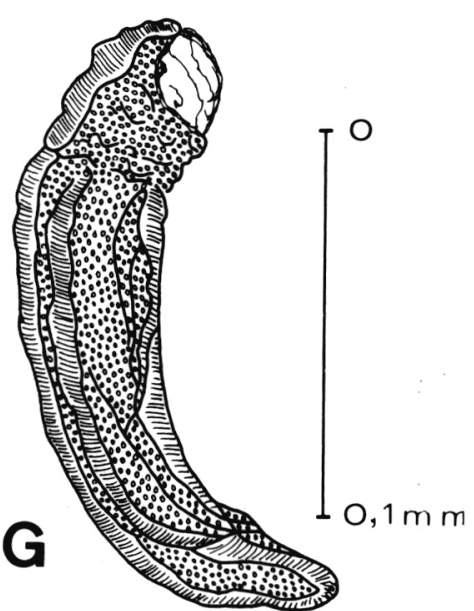


MÂlE (fig. 1)

Ailes latérales longues, se continuant sur les flancs latéraux de la bourse caudale. Bourse caudale avec des lobes subégaux. Neuf paires de papilles caudales, quatre précloacales et cinq postcloacales, spicules très inégaux. Gubernaculum fortement sclérotisé et bien développé. Le spicule gauche est long et présente sur l'extrémité proximale une poignée moruliforme, l'extrémité distale est terminée en pointe. Le spicule droit est plus court et trapu. Cloaque à peine saillant avec un rebord cuticulaire crénelé caractéristique.

Dimensions : o holotype et 9 or paratypes obtenus de Pie (tableau I).

\section{Femelle (fig. $2 A, B, C, D$ et $F$ )}

Les ailes latérales se terminent à la moitié du corps. Vulve simple située dans la moitié postérieure du corps. Ovéjecteur très long dirigé en avant. Anus à peine saillant. Queue courte avec deux phasmides. Ovipares. OEufs embryonnés.

Dimensions : $\subsetneq$ allotype et 9 paratypes obtenus de Pie (tableau I).
OEufs (fig. 2 E)

Étudiés à partir de fragments utérins d'une des femelles. Ils sont embryonnés dans l'ovéjecteur. Les extrémités antérieure et postérieure de la larve sont arrondies. Dans la tête de la larve, on peut distinguer une structure triradiaire très réfringente.

Dimensions sur un échantillon de 30 œufs. De 0,041 à $0,042 \mathrm{~mm}(\mathrm{X}=0,041)$ pour 0,026 à $0,028 \mathrm{~mm}(\mathrm{X}=0,027)$.

\section{DISCUSSION}

Diverses études taxonomiques réalisées jusqu'à maintenant (Skrjabin, 1971; Chabaud, 1959; Popova, 1966 et Chabaud, 1975), ont souligné comme un caractère différentiel et remarquable pour la famille Gongylonematidae, la présence d'un nombre plus ou moins grand d'épaississements verruqueux dans la région antérieure du nématode, dont la distribution et la morphologie sont utilisées par les différents auteurs pour la description des espèces et pour la réalisation de clés, comme la proposition de Barre (1980) pour la détermination des espèces de gongylonèmes

Tableau I. - Dimensions en millimètres.

\begin{tabular}{|c|c|c|c|c|c|c|c|c|}
\hline \multirow[b]{2}{*}{ Paramètres } & \multirow[b]{2}{*}{ Holotype } & \multirow[b]{2}{*}{ Allotype } & \multicolumn{3}{|c|}{ Paratypes $९$} & \multicolumn{3}{|c|}{ Paratypes $\mathrm{O}^{\prime}$} \\
\hline & & & Min. & Max. & $X$ & Min. & $\operatorname{Max}$ & $X$ \\
\hline Longueur & 14,80 & 27,40 & 26,20 & 30,80 & 28,44 & 10,80 & 17,60 & 15,42 \\
\hline $\begin{array}{l}\text { Largeur au niveau de : } \\
\text { - pharynx } \\
\text { - l'œsophage } \\
\text { - l'anus } \\
\text { - la vulve } \\
\text { - la cloaque }\end{array}$ & $\begin{array}{c}0,057 \\
0,239 \\
- \\
\overline{-}, 239\end{array}$ & $\begin{array}{c}0,062 \\
0,330 \\
0,119 \\
0,282 \\
-\end{array}$ & $\begin{array}{l}0,057 \\
0,263 \\
0,119 \\
0,287 \\
-\end{array}$ & $\begin{array}{l}0,067 \\
0,325 \\
0,143 \\
0,330 \\
-\end{array}$ & $\begin{array}{l}0,060 \\
0,332 \\
0,130 \\
0,304 \\
-\end{array}$ & $\begin{array}{c}0,047 \\
0,186 \\
- \\
\overline{0} \\
0,191\end{array}$ & $\begin{array}{c}0,062 \\
0,273 \\
- \\
\overline{-} \\
0,296\end{array}$ & $\begin{array}{c}0,052 \\
0,243 \\
- \\
\overline{-} \\
0,264\end{array}$ \\
\hline Largeur de l'aile latérale & 0,009 & 0,022 & 0,016 & 0,024 & 0,021 & 0,011 & 0,014 & 0,011 \\
\hline $\begin{array}{l}\text { Dist. de l'apex : } \\
\text { - deirides } \\
\text { — aile latérale } \\
\text { — anneau nerveux } \\
\text { - pore excréteur }\end{array}$ & $\begin{array}{l}0,186 \\
0,249 \\
0,311 \\
0,445\end{array}$ & $\begin{array}{l}0,185 \\
0,257 \\
0,371 \\
0,574\end{array}$ & $\begin{array}{l}0,154 \\
0,277 \\
0,383 \\
0,550\end{array}$ & $\begin{array}{l}0,282 \\
0,364 \\
0,479 \\
0,632\end{array}$ & $\begin{array}{l}0,228 \\
0,315 \\
0,442 \\
0,571\end{array}$ & $\begin{array}{l}0,142 \\
0,164 \\
0,239 \\
0,340\end{array}$ & $\begin{array}{l}0,225 \\
0,287 \\
0,383 \\
0,502\end{array}$ & $\begin{array}{l}0,193 \\
0,249 \\
0,327 \\
0,436\end{array}$ \\
\hline $\begin{array}{l}\text { Dist. de l'extrémité caudale : } \\
\text { — vulve }\end{array}$ & - & 5,80 & 5,20 & 6,90 & 6,32 & - & - & - \\
\hline Dist. deiride-aile latérale & 0,063 & 0,072 & 0,065 & 0,124 & 0,087 & 0,022 & 0,077 & 0,055 \\
\hline Cavité buccale $(\mathrm{L} \times \mathrm{A})$ & $5 \times 25$ & $7 \times 21$ & $5 \times 22$ & $7 \times 24$ & $6,1 \times 22,88$ & $3 \times 24$ & $5 \times 27$ & $4,7 \times 23,4$ \\
\hline OEsophage musculaire $(\mathrm{L} \times \mathrm{A})$ & $550 \times 19$ & $684 \times 23$ & $646 \times 28$ & $795 \times 33$ & $708,4 \times 27,7$ & $383 \times 16$ & $641 \times 23$ & $571,5 \times 23$ \\
\hline CEsophage glandulaire $(\mathrm{L} \times \mathrm{A})$ & $3650 \times 110$ & $4440 \times 145$ & $4225 \times 138$ & $5458 \times 136$ & $4902,8 \times 144$ & $2217 \times 74$ & $4159 \times 110$ & $3566,7 \times 116$ \\
\hline Spicule gauche $(\mathrm{L} \times \mathrm{A})$ & $1077 \times 19$ & - & - & - & - & $1197 \times 14$ & $1335 \times 23$ & $1245,7 \times 19,7$ \\
\hline Spicule droit $(\mathrm{L} \times \mathrm{A})$ & $205 \times 71$ & - & - & - & - & $215 \times 52$ & $249 \times 71$ & $230,5 \times 62,8$ \\
\hline Gubernaculum $(\mathrm{L} \times \mathrm{A})$ & $129 \times 23$ & - & - & - & - & $119 \times 14$ & $121 \times 25$ & $115,6 \times 23,7$ \\
\hline CEufs $(\mathrm{L} \times \mathrm{A})$ & - & $42 \times 28$ & $41 \times 26$ & $42 \times 28$ & $41,44 \times 27,44$ & - & - & - \\
\hline Queue $(\mathrm{L} \times \mathrm{A})$ & $675 \times 239$ & $314 \times 121$ & $242 \times 135$ & $335 \times 129$ & $292,5 \times 129,4$ & $359 \times 191$ & $766 \times 296$ & $649,7 \times 264,5$ \\
\hline
\end{tabular}

$\mathrm{X}=$ moyenne $; \mathrm{L}=$ longueur $; \mathrm{A}=$ largeur. Les mesures s'expriment en millimètres, sauf celles $(\mathrm{L} \times \mathrm{A})$ en micromètres. 
d'oiseaux domestiques et sauvages. Par ailleurs, Chabaud (1975) indique que la classification des spiruridés est essentiellement fondée sur les structures céphaliques. Les structures céphaliques des nématodes décrits dans ce travail, caractérisées par la présence de deux élévations dentiformes sclérotisées (l'une dorsale et l'autre ventrale), trois paires de papilles labiales internes disposées dans deux groupes latéraux, quatre papilles labiales externes disposées dans les angles du cadre buccal saillant, indiquent clairement leur appartenance à la famille Gongylonematidae, et au genre Gongylonema (unique genre de la famille).

La classification du genre Gongylonema en deux sous-

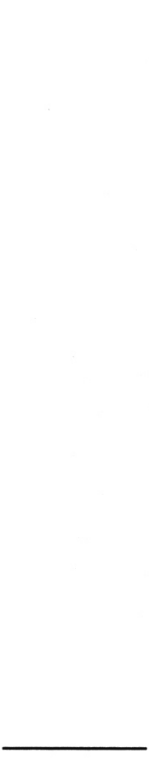

FIG. 2. - Gongylonema (Progongylonema) pacoi n. sp. Femelle.

A : extrémité antérieure. B : détail de la cavité buccale. C : extrémité céphalique, vue apicale. D : extrémité postérieure. E : œuf. F : détail de la vulve.

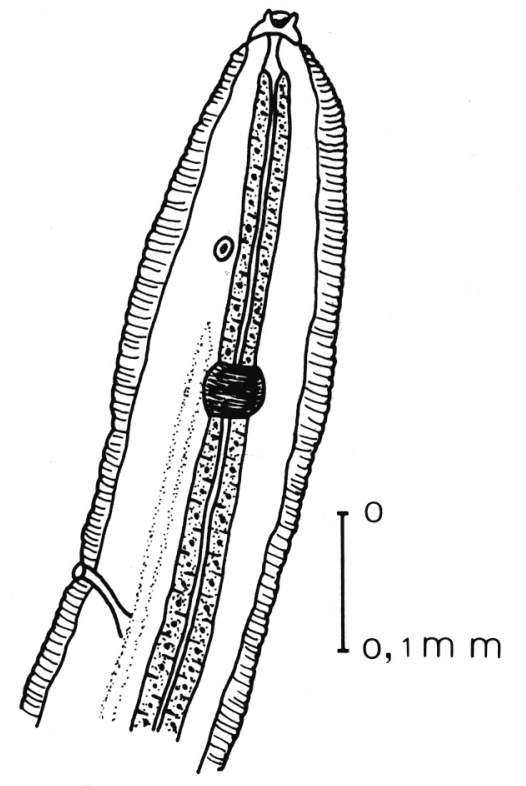

A

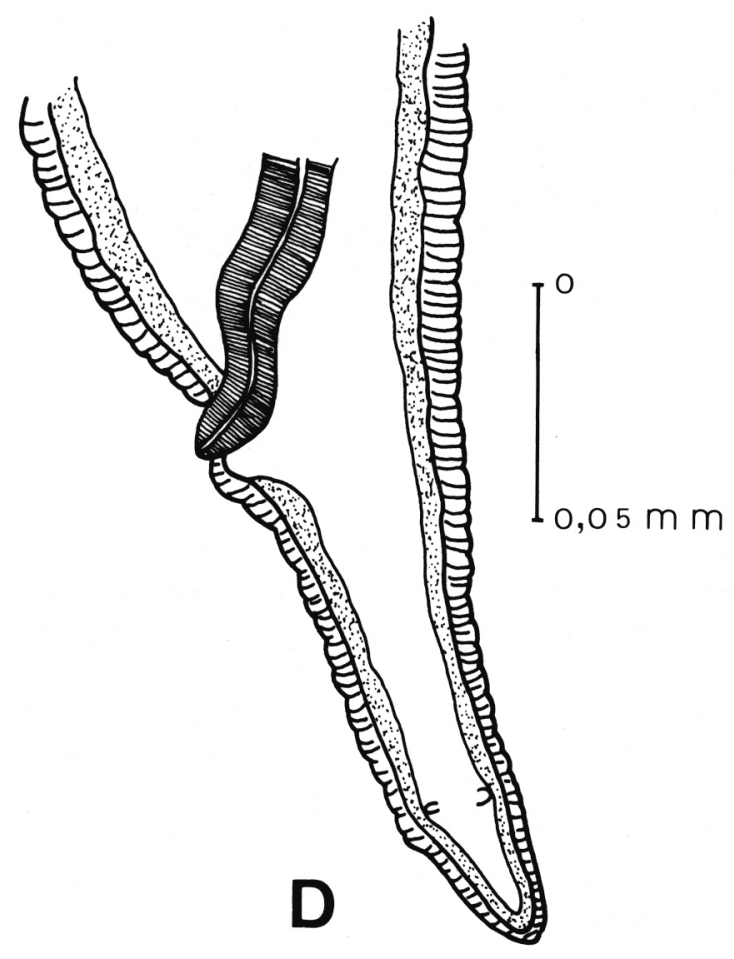

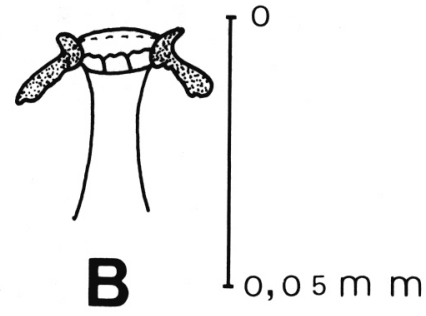
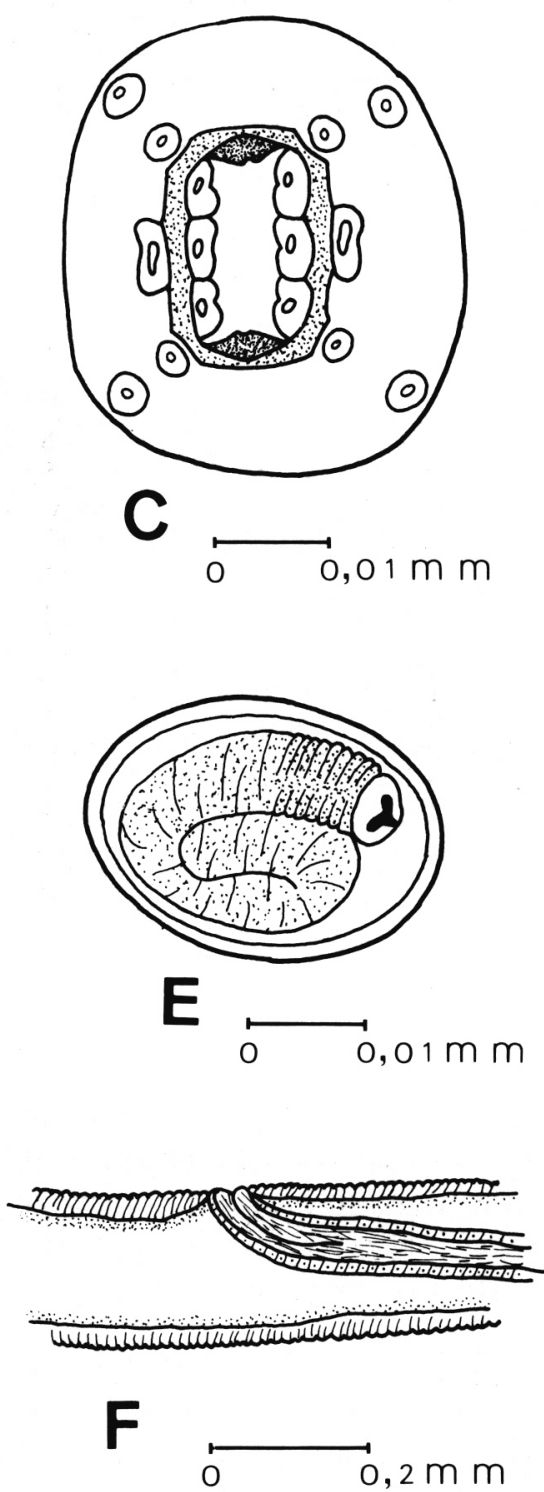

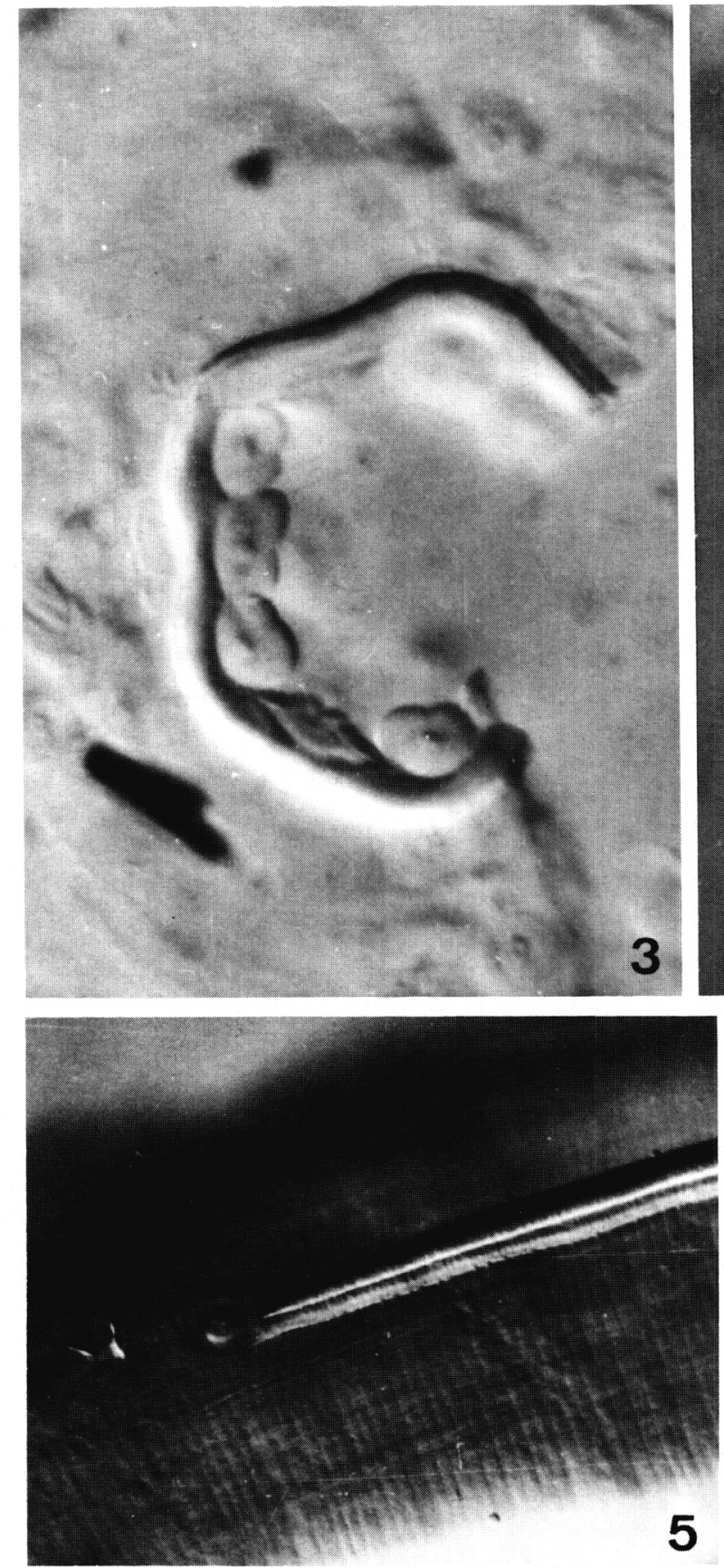

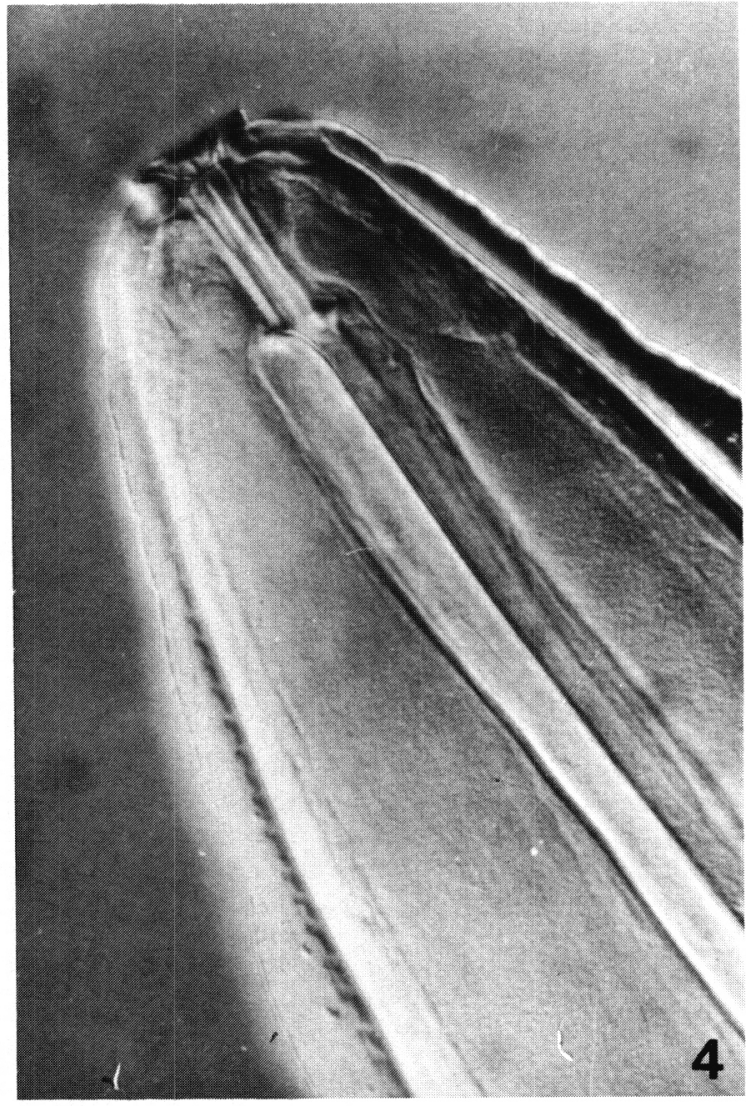

FIG. 3-5. - Gongylonema (Progongylonema) pacoi n. sp. Paratype. Microphotographies en contraste interférfentiel de Nomarski.

3. - Extrémité céphalique en vue apicale.

4. - Extrémité antérieure en vue latérale.

5. - Détail de la deiride, des ailes latérales et des stries cuticulaires. genres (Chabaud, 1975) se fonde sur le fait que les Gongylonema (Gongylonema) (Molin, 1857) Chabaud, 1975, présentent de nombreux écussons cuticulaires, des spicules très inégaux, avec un gubernaculum; ils sont parasites de mammifères et d'oiseaux tandis que les Gongylonema (Gongylonemoides) (Lent et Freitas, 1937) Chabaud, 1975, présentent de rares écussons cuticulaires, des spicules subégaux, avec absence de gubernaculum et sont parasites de marsupiaux. Le nématode que nous avons décrit dans ce travail est différencié morphologiquement de Gongylonema (Gon- gylonema) par le manque d'écussons cuticulaires et biologiquement parce qu'il est parasite de Corvidae et de Passeriformes chez lesquels il est localisé dans la muqueuse du bec. Jusqu'à maintenant, la plupart des espèces des Gongylonèmes d'oiseaux ont été trouvées principalement chez les Galliformes et rarement chez les Falconiformes (Gongylonema falconis), Charadriiformes (Gongylonema metopidius) et Anseriformes (Gongylonema congolense) et toujours dans la muqueuse du jabot et de l'œsophage. Gongylonema congolense a été mentionné une fois dans la muqueuse du bec 
(cit. Skrjabin, 1971). Les différences avec Gongylonema (Gongylonemoides) sont évidentes aussi, car ils ne partagent aucune des caractértistiques mentionnées dans ce sousgenre. C'est pourquoi nos exemplaires partagent des ressemblances et des différences avec Gongylonema (Gongylonema) et ne comportant que des différences avec Gongylonema (Gongylonemoides), les parasites décrits dans ce travail caractérisés par l'absence totale d'écussons cuticulaires, ne peuvent pas être de ce fait assignés dans aucun des sous-genres précédemment mentionnés. Pour cette raison, nous proposons la création d'un nouveau sous-genre Gongylonema (Progongylonema) dont les caractéristiques morphologiques sont celles de cette nouvelle espèce choisie pour type.

L'espèce type Gongylonema (Progongylonema) pacoi est dédiée à notre ami et camarade Paco Martinez, Professeur de Parasitologie Vétérinaire de la Faculté de Médecine vétérinaire de Cordoue, récemment décédé.
Remerciements. - Ce travail fait partie du projet de Recherche «Parásitos y Medio Ambiente » de la Consejeria de Educación y Ciencia de la Junta de Andalucia. Nous voulons remercier Mme Teresa Medina pour son assistance comme secrétaire.

\section{RÉFÉRENCES}

Barré N : Gongylonema grabei (Spiruroidea: Gongylonematidae), parasite nouveau de Gallus gallus domesticus, Linné à la Réunion. Rev. Elev. Med. Vet. Pays Trop., 1980, 33, 385-391.

Chabaud A. G. : Phénomène d'évolution régressive des structures céphaliques et classification des nématodes spiruroidea. Parassitologia, 1959, 1, 11-20.

Chabaud A. G. : Keys to the nematode parasites of vertebrates. Anderson, Chabaud and Willmott. Eds. No. 3 C. A. B. Farnham Royal, Bucks (England), 1975.

Popova J. G. : Morphological and taxonomic study of nematodes of the subfamily Gongylonematinae. Veterinariya, Kiev, 1966, 6, 121-138.

Skrjabin K. I., Sobolev A. A., Ivashkin V. M. : Spirurata of animals and the diseases caused by them. Vol. 4, Thelazioidea. I. P. S. T., 1971, Jerusalen. 